\title{
Corridors for Robot Team Navigation
}

\author{
Zack Butler and Carlos Bribiescas
}

Dept. of Computer Science

Rochester Institute of Technology

Rochester NY USA

zjb@cs.rit.edu, cab3596@rit.edu

Summary. In previous work, we proposed the use of virtual corridors to move a group of agents (animals) from one location to another. Using virtual fence technology, we can create a corridor from a set of fences to generate the desired gross motion of the herd without overspecifying paths for the animals. This allows us to reduce the amount of stimulus given to the animals while allowing them to naturally avoid obstacles (or other agents) without having to consider them during planning. In addition, a single corridor can be used by the entire group, simplifying planning and requiring only limited communication. In this work, we have implemented virtual corridors for robot team navigation, both in simulation and on two Pioneer mobile robots, and performed a number of experiments with two aims in mind. First, we wish to determine if, and under what circumstances, corridors are a practical solution to navigation by a team of robots. Secondly, by investigating different underlying motion algorithms for the robots, we may be able to suggest how successful corridors can be for different systems (including animals). We show success in open environments over a variety of experiments, with varying efficiency from the different motion models, and partial success among complex obstacles and with teams of up to five robots using a single corridor simultaneously.

\section{Introduction}

Unlike robots, animals have innate navigational and obstacle-avoidance capabilities. However, unlike animals, robots will (generally) go where they are told. In our past work [3], we developed the idea of virtual fences to bring some level of path planning and control to the animal systems. That is, by putting a device on the animal's collar which can detect the animal's position, we can define a virtual fence so that the collar induces a stimulus on the animal when it crosses this fence, thus directing the animal to remain in a predefined area. The positive effect of such stimuli has been verified in [7]. In addition, since the collar device includes both GPS and computation, it is easy to make the virtual fences move over time to move the animals as desired. Finally, it is also possible to use the same set of virtual fences for all the animals in a 
herd, greatly simplifying the planning for this cooperative system, leveraging the animals' local navigation capabilities and inducing stimuli only to achieve global path goals.

In this work, we are interested in looking at the problem from a robotics viewpoint. That is, can robots with simple obstacle-avoidance capability use the same type of virtual fences to induce path following in a simple way? This would provide several avenues of simplification: a single plan can be generated and used for a team of robots to get from one area to another, dynamic obstacles may be implicitly handled within the corridor, and the robots do not need to able to communicate with or explicitly recognize each other to execute the path. We are also interested in the level of innate navigational ability required to successfully follow the desired path - is a random walk sufficient, or are maps or state-based planning necessary? Our technique requires a base map to develop the corridors, but the width of the corridors allows the robots to deal with small unmapped obstacles and dynamism of the environment with no additional overhead.

A large amount of work has been done in cooperative robotics in terms of multi-robot path planning. This can take the form of a single plan for all robots, essentially forming a plan in a $2 n$ dimensional space for $n$ robots that handles collision avoidance by adding collisions as obstacles in the configuration space (e.g. [9]). Alternately, each robot can make a plan independently, with distributed traffic control used to prevent collision and deadlock (e.g. [5]). These plans can handle the case of robots with qualitatively different goal locations, but may not be suited to dynamic environments as we expect to encounter here. For robots traveling together, formation control can be used, in which a leader robot has a path to follow and the others take a position relative to the leader. With appropriate control policies, this has been shown to be robust even in the presence of obstacles that force the robots out of formation $[1,4]$. However, the role of each robot within the formation must be specified and possibly reassigned in the case of failure. In the algorithm used here, a single plan used is homogeneously across all robots in the system. Explicit inter-robot sensing and/or communication can also be used to improve navigation during path execution [8]. In contrast, our work does not rely on such sensing, and explores what can be accomplished when other robots are treated as (dynamic) obstacles within a single planned path.

\subsection{Corridor planning}

Using dynamic virtual fences, we have previously developed a planning algorithm [2] that creates a virtual corridor through the environment to herd a group of agents from one given area to another. The underlying motivation is twofold. First, that having the majority of the virtual fences fixed over time (as opposed to a moving rectangle that contains the group) is both simpler to create and it would seem more intuitive for the agents to follow. Second, the corridors can be embedded in the natural obstacles of the environment, so 


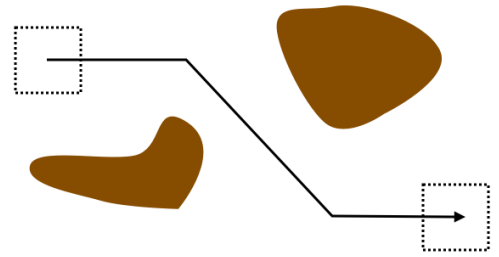

(a)

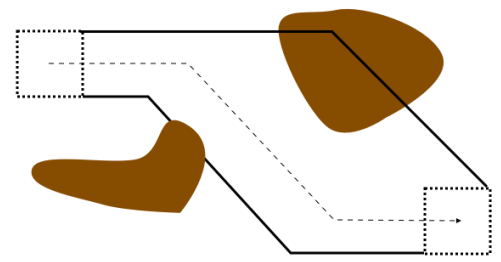

(c)

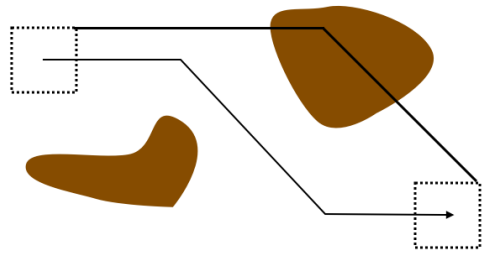

(b)

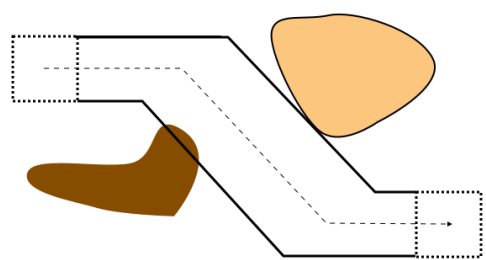

(d)

Fig. 1. Example of corridor creation. (a) A path is planned for a single point. (b) The path segments are extended in one direction to form corridor walls, going through an obstacle in the environment. (c) The expansion on the other side is stopped to avoid creating a dead end in the corridor. (d) If an obstacle is only algorithmic, that is, the corridor must keep the agents out of the given area, this can also be handled during the expansion as shown.

that where possible, the visible features are used to control the agents rather than invisible ones. This latter concept also can allow corridors with simpler shape as they can go straight through obstacles.

The process to create a corridor in a sample environment is given in Fig. 1. First, a path is first created for a single point using a straightforward A* implementation. This is initially limited to travel along the eight cardinal directions, and the path costs used by $\mathrm{A}^{*}$ are chosen such that the path will have as few turns as possible, so that the resulting corridors will have fewer segments. Each segment of this point-based path is then moved perpendicularly in both directions to create a corridor segment. Care is taken to avoid introducing local dead-ends in the corridor. We can also specify areas which the corridor must not contain (such as a set of stairs that the robot cannot sense), and the expansion process will simply stop at those points. We then create a single moving wall that travels along the length of the corridor to keep the agents moving toward the goal area. The moving wall is oriented perpendicularly to the original path for each segment except at corners, where it pivots around the vertex at the inside of the corner. For a full description of this technique, see [2]. 


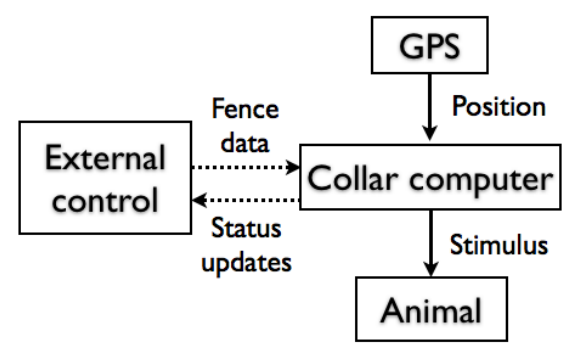

(a)

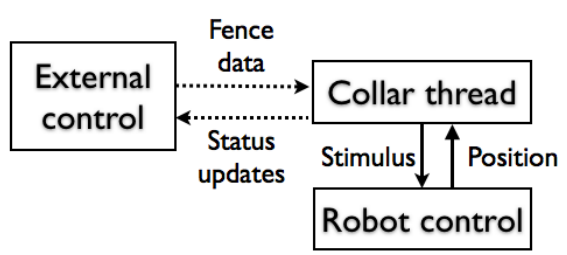

(b)

Fig. 2. Block diagram of collar architecture. (a) In the animal system and (b) in an indoor robot system. In both cases, external control communicates with onboard computing intermittently through a wireless connection (dashed lines), while fencebased control takes place in real time on the collar (solid lines). In the robot, both robot control (random walk) and collar control operate on the same computer, but in separate threads.

\section{System Architecture}

In the animal implementation of the virtual corridors, each animal is given a collar with GPS, computation, wireless communication and a stimulus device. At user-defined intervals, a set of virtual fences is uploaded to the animals, and these fences are then enforced by the computer via the stimulus device. It is usually the case that all the animals in a group will get the same fences, though this is not required. The fences may move according to a schedule to enable mustering from one location to another, or may simply be fixed as normal fences. A block diagram of this system can be seen in Fig. 2a.

To implement this on robots, we simply replace the animal with a robot that is programmed to move about and react to "stimulus" (whatever that may mean in the context of the implementation). We have implemented this with a multi-threaded client program in the Player/Stage framework [6]. One thread runs simple motion control that communicates via the Player interface with the robot hardware (or Stage simulation). A second thread runs the collar control program which obtains fences from a data file. A shared data object allows for stimuli to be sent from the collar to the robot. For the case of indoor mobile robots, we must also find an alternative to GPS. For simplicity in this case, as seen in Fig. 2b, we have used dead reckoning, with another shared data object sending robot positions from the motion control thread to the collar thread. We note that since Player/Stage simulates perfect dead reckoning, the simulation is more equivalent from a functionality standpoint to the GPSbased outdoor case than the indoor case even though the implementation is the same as is used indoors. 


\section{Motion models}

As mentioned previously, an important facet of this work is the evaluation of different underlying robot motion behaviors for use with the virtual corridors. These include simple random walks with varying parameters as well as the use of wandering with additional state.

The simplest motion model used is a safe random walk. In this model, the robot first checks that no obstacle is within approximately one robot diameter ahead of it, and if such an obstacle is present, it stops forward progress and turns toward the side with greater clearance. Otherwise, it uses its four forward-facing sonar sensors (covering approximately $\pm 30^{\circ}$ ) to set its forward velocity. It averages the distances of these sensors and divides the average distance by a speed factor (essentially setting a time-to-collision). For our robots, the sonar readings are capped at $5 \mathrm{~m}$, so a speed factor of 10 corresponds to a maximum velocity of $0.5 \mathrm{~m} / \mathrm{s}$. It then chooses a random angular velocity and executes the motion. In these experiments we use a uniformly random angular velocity between -0.5 and $0.5 \mathrm{rad} / \mathrm{s}$, though we have also tested other models with similar results.

In addition to the unconstrained motion, there are different ways in which the robot can react to a stimulus, even within the domain of attempting to model likely animal behavior. Inspired by the animal hardware, the stimulus itself is simply bilateral, that is, it can either be on the right or left of the robot but with no additional direction or magnitude information. The robot will then react to the stimulus by turning away from it by a predetermined amount - that is, if the robot drives forward into a right-side virtual fence, it will receive a stimulus on the right, and thus turn toward the left. In this work, we have used responses of a constant turn of $90^{\circ}$, a constant turn of $45^{\circ}$, and a uniformly random turn between $45^{\circ}$ and $135^{\circ}$.

Going up a step in complexity, the robots can keep some state about the stimuli they have received. As a first approach, we have chosen to keep a "drift" vector $d$ that remembers in what direction stimuli has been received and decays over time. Each time a stimulus is felt, the robot computes an aversion direction perpendicular to its current direction of travel and adds this aversion (with magnitude 0.25) to $d$. The magnitude of $d$ is capped at 0.5 to ensure stable behavior. Each time step that no stimulus is felt, the robot computes a random vector $r$ with magnitude 0.5 based on the random walk used above, and the vector $d+r$ is multiplied by the nominal speed to determine the velocity of travel (note that the magnitude limits mean that the nominal speed will never be exceeded). In addition, the magnitude $d$ is decreased by .0075 per second to simulate the slow forgetting of stimuli in the past (and allow for virtual walls in different directions to be handled more effectively). 


\section{Experimental results}

We ran a number of experiments in different environments to attempt to determine the utility of corridor planning. These included varying the motion model, both qualitatively and quantitatively, and the speed of the back wall of the corridor. The majority of the experiments were done with two robots sharing a single corridor, though we also performed some tests with five robots to explore the parallelism available. We defined success as whether the robots reached the last 2 meters (of the $>25 \mathrm{~m}$ corridor) before the back wall reached the end of the corridor.

The first set of experiments was designed to determine which stimulus response would be most effective in the context of the corridors. We tested a straight empty virtual corridor with the velocity of the moving back fence $\left(v_{f}\right)$ between $0.02 \mathrm{~m} / \mathrm{s}$ and $0.1 \mathrm{~m} / \mathrm{s}$ and robot speed factors $(s f)$ of 10 and 20 (recall that since this number divides the sonar reading to obtain the forward velocity, a larger $s f$ indicates slower nominal velocity). Over all the stimulus responses, we found that the fence velocity had only a moderate effect on the time taken to travel the corridor, but a larger effect on the frequency of successful completion of the corridor. For the $90^{\circ}$ response, for example, the robots were able to travel the corridor in all trials with $s f=10$ and $v_{f}=0.02$ and 0.04 , and in these two cases, the time taken to reach the end of the corridor was $199.5 \mathrm{~s}$ and $189.5 \mathrm{~s}$ respectively. As the fence velocity increased, the success rate decreased, with $16 / 20$ trials succeeding at $v_{f}=0.08$ and only $5 / 20$ at $v_{f}=0.1$. In this last case, when the robots did succeed, they did so in an average of $138.7 \mathrm{~s}$, somewhat faster than with the slower fence, as expected. The random-turn stimulus response had similar rates of success at the higher robot velocity, but with significantly longer time taken to travel the corridor $\left(294 \mathrm{~s}\right.$ at $v_{f}=0.02$ and $301 \mathrm{~s}$ at $\left.v_{f}=0.04\right)$. At the slower robot velocity and/or higher fence velocity, the success rate was significantly lower than the $90^{\circ}$ response, with only $2 / 20$ completing at $v_{f}=0.1$ and $s f=10$. Finally, for the $45^{\circ}$ response, we found higher success rates (100\% success at $f_{v}=0.08, s f=10$ and $\left.v_{f}=0.04, s f=20\right)$ and faster traversal times $(156.1 \mathrm{~s}$ at $f_{v}=0.02$ and $144.8 \mathrm{~s}$ at $\left.f_{v}=0.04\right)$ than either of the other responses. Our observations are that the long and narrow aspect of the corridor rewards smaller turns that tend to point the robot along the corridor rather than back across it.

We also analyzed the number of stimuli that had to be applied to get the robots to reach the end of the corridor in each case. Here we found correlation between the amount of stimulus and the time to completion. For the $90^{\circ}$ response and the $45^{\circ}$ response, stimulus levels were quite similar, averaging approximately 20 total stimuli appiled to the two robots for the velocities with $100 \%$ success, while the random-angle response required significantly more stimulus, approximately 40-45 per run under the same conditions.

Using the $45^{\circ}$ stimulus response with $s f$ fixed at 10 , we then tested a variety of other environments to see how well it can move teams through 


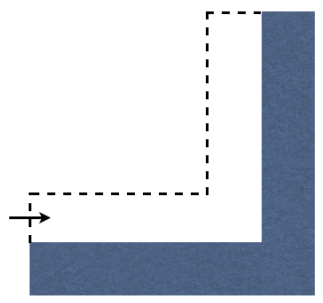

(a)

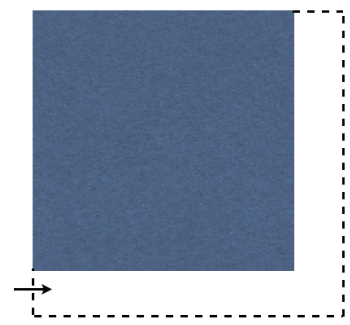

(b)

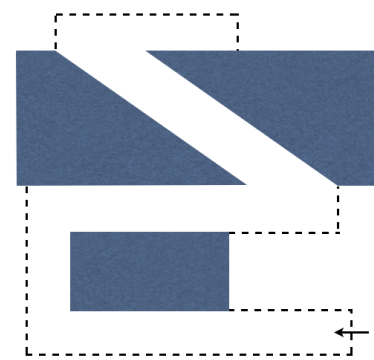

(c)

Fig. 3. Environments used in simulation experiments: (a) "L" test (b) "Square" test (c) "Complex" test. Solid shapes represent physical obstacles and dashed lines represent virtual fences. The arrow indicates the moving back wall of the corridor (shown at its initial configuration. Robots start in a group near the back wall. All environments are between 25 and 30 meters in width.

areas of greater complexity. These environments are shown in Fig. 3. On the "L" test, the robots completed the corridor in $100 \%$ of tests up to $f_{v}=0.06$, and $90 \%$ of tests at $f_{v}=0.08$ and 0.1 . Stimulus levels were actually lower than those in the blank corridor, since the real wall acts as one side of the corridor and can be avoided without external stimulus.

The "square" and "complex" tests, on the other hand, are somewhat more challenging. Depending on the heading of the robot when it hits the moving fence, the stimulus may direct it toward an obstacle, and the robot will not be able to escape both the virtual and physical walls. This is especially pronounced in the complex test, where the moving back wall meets some obstacles at an acute angle. Also, even though the robots are fast enough to outrun the back wall, around the corners of obstacles, when the back wall turns to follow the direction of the corridor, it may actually provide a stimulus toward the previous segment of the corridor depending on the robot's heading. We also saw occasional livelock when the two robots would meet at certain angles near a wall due to the simple obstacle avoidance. In the square tests, we obtained success rates of about $80-90 \%$ for all fence velocities, while in the complex test, there was a more pronounced dependence on $f_{v}$. At $f_{v}=0.02,9$ of 21 trials succeeded, though in another 8 trials, one of the two robots made it to the end of the corridor. At $f_{v}=0.04,7$ of 21 trials were successful, but only 3 at $f_{v}=0.06$. In all case, in the trials that did succeed, stimulus levels remained low, since a large fraction of each corridor is defined by physical features.

We also tested five robots in the some of the same corridors. In these cases, the implicit traffic control gets a much more difficult test, as the virtual corridor is less than 10 robot widths across. As such, the success rates go down and the traversal times increase compared to the two-robot case. However, the algorithm still produces good results. In the blank corridor, an average of at 
least 4.8 out of the 5 robots reached the end in the allotted time for all fence speeds up to $0.08 \mathrm{~m} / \mathrm{s}$, though with completion times ranging from $362 \mathrm{~s}$ to $489 \mathrm{~s}$, approximately three times as long as two robots in the same size corridor. The amount of stimulus required was slightly higher even on a perrobot basis, averaging between 61.3 and 77 total events per run. Similarly, the "L" test took three to four times as long as the two-robot case and with 4.5 robots or better finishing the corridor at $f_{v}=0.02$ and 0.04. In the "complex" test, with narrow physical as well as virtual corridors, an average of 3.5 and a minimum of 2 of the 5 robots finished each trial at $f_{v}=0.01$.

Finally, we tested the drift-based control in the same environments to judge whether maintaining additional state can improve the efficiency of using the corridors. We retained the $45^{\circ}$ stimulus response for direct comparison to the stateless model. For the blank corridor, we found significant performance improvements. Specifically, the robots were $100 \%$ successful at even higher fence speeds (up to $f_{v}=0.1$ at $s f=10$ and $f_{v}=0.06$ at $s f=20$ ) than any of the previous motion models. These are achieved with approximately half the number of stimuli used in the stateless case, and with slightly lower traversal times. With five robots in this corridor, similar success rates are seen as with the stateless case, but again at higher $v_{f}$, with an average of at least 4.5 robots reaching the end of the corridor up to $v_{f}=0.1$. In contrast, the success rates in the other corridors were significantly lower than with the stateless technique. In the square corridor, $90 \%$ of trials succeeded at $f_{v}=0.02$, but only about $50 \%$ at $f_{v}=0.04$ and 0.06 . In the complex corridor, only about $30 \%$ of the time did even one of the two robots finish. Our observations were that even with the decaying drift vector, when the robot came to the corner of two virtual fences, it would often travel directly into the far fence, and end up with a drift parallel to the corridor wall. The drift also exacerbated the problem of the virtual wall conflicting with the physical wall, as the effect of the stimulus against the physical wall would linger.

\subsection{Hardware experiments}

To test the viability of the algorithm in hardware, we implemented corridor following on our two Pioneer P3-DX robots. Each robot has eight forwardfacing sonar sensors as in our simulations, and only dead reckoning was used for positioning, as in Fig. 2b. Two environments were tested, one in open space and the other along a hallway.

In the first set of experiments, a virtual corridor of length $15 \mathrm{~m}$ and width $1.8 \mathrm{~m}$ was used within the atrium of our building. There were no permanent obstacles within the virtual corridor. We ran five trials in which the two robots were started at the same time and allowed to run down the corridor. In all cases, both robots successfully reached the other end of the atrium. Dead reckoning error meant that their actual positions were occasionally outside the intended corridor, but never by far enough to cause collisions or confusion 
on the part of the robots. Additionally, because of the nature of the algorithm, occasional intrusions into the corridor by passers-by were also handled successfully without replanning.

The second set of experiments was run in a hallway of width $2.1 \mathrm{~m}$ and length $20 \mathrm{~m}$ in which the virtual corridor was wider than the hallway. Thus, only the moving back wall was used to herd the robots down the hall. In one of the five experiments run, both robots were able to reach the end of the hallway. In the other four, one of the robots was able to travel the entire hallway, while the other did not. The effect looked to be similar to the simulations in which the back wall and physical wall give contradictory forces, however in this case it also seemed that the limited angular acceleration of the hardware caused a slower stimulus response than in simulation and made it more difficult to escape the back wall once it caught up to the robot.

\section{Conclusions}

Overall, the work here has shown that the current implementation of virtual corridors can be used for path execution simultaneously by a team of robots, both simulated and in hardware, with greatest success in open environments. We found significant differences in the results with different stimulus response behaviors, with a smaller turn being more effective than a larger or random turn. Using the most effective behavior, the robots are able to avoid each other and small obstacles while reaching the end of the corridor. In simulated environments without obstacles (with respect to the virtual corridor), the robots completed their mission every time as long as the fence speed was less than $15 \%$ of the nominal robot speed, and environments with few obstacles, even large ones, could be traversed most of the time. The most complex environment tested could be handled with moderate success. Adding a small amount of state to the motion control improved the success rates and efficiency in straight corridors, but decreased performance in complex environments. Future work will entail investigating other ways to incorporate stimuli into memory to improve efficiency in more environments, especially to handle situations where the virtual fences and physical obstacles give contradictory impulses.

We are also working on extending the control algorithm to include herding behaviors, that is, robots will be attracted to each other in the same way that herd animals are by nature. This will require some type of mutual sensing, or at least communication of positions, but may allow easier recovery from leaving the corridor or falling behind the moving fence as the robot outside the corridor will see the other robots still within it. Other animal traits such as leading and following can also be tested to judge their effects (either positive or negative) under corridor control.

On the other hand, to make this algorithm more suitable to the pure robotics context, we can alter both the corridor execution as well as the con- 
trol. On the corridor side, we may be able to improve performance in complex environments and with the hardware (at the expense of execution speed) by using a ratcheting back wall instead of the constant-speed wall. That is, the back wall will move up as far as the last robot in the corridor, but not beyond, so that this robot can catch up to the group. This will also require communication between robots to keep the back wall the same within the herd, and may be less necessary if the herding behavior is used. Without adding communication, we can still fold in simple localization techniques and test these on the indoor hardware. In the obstacle-rich indoor environment, we may be able to specify virtual fences with respect to known physical obstacles, thus circumventing the localization problem. This would be similar to topological path planning and execution, but with the added benefit of shared corridors for efficient team navigation without explicit mutual sensing.

\section{Acknowledgments}

This work was supported in part by NSF grant IIS-0513628.

\section{References}

1. E. Bicho and S. Monteiro. Formation control for multiple mobile robots: a nonlinear attractor dynamics approach. In Proc. of IEEE IROS, pages 2016-22, 2003.

2. Z. Butler. Corridor planning for natural agents. In IEEE International Conference on Robotics and Automation, 2006.

3. Z. Butler, P. Corke, R. Peterson, and D. Rus. From robots to animals: Virtual fences for controlling cattle. Int'l Journal of Robotics Research, 25(5):485-508, 2006.

4. J. Desai, J. Ostrowski, and V. Kumar. Modeling and control of formations of nonholonomic mobile robots. IEEE Trans. on Robotics and Automation, 17(6):905-8, 2001.

5. D. D. Grossman. Traffic control of multiple robot vehicles. IEEE Journal of Robotics and Automation, 4(5):491-7, 1988.

6. The player project. http://playerstage.sourceforge.net.

7. T. M. Quigley, H. R. Sanderson, A. R. Tiedemann, and M. K. McInnis. Livestock control with electrical and audio stimulation. Rangelands, pages 152-5, June 1990 .

8. A. Sanderson. A distributed algorithm for cooperative navigation among multiple mobile robots. Advanced Robotics, 12(4):335-49, 1998.

9. L. Whitcomb, D. Koditschek, and J. Cabrera. Toward the automatic control of robot assembly tasks via potential functions: The case of 2-d sphere assemblies. In Proc. of IEEE ICRA, pages 2186-91, 1992. 